\title{
Modelling Various Solar Cells Materials Using Lorentzian-Drude Coefficients
}

\author{
https://doi.org/10.3991/ijes.v4i4.6566 \\ S. Abdellatif, ${ }^{1}$ K. Kirah, ${ }^{2}$ R. Ghannam, ${ }^{3}$ A. S. G. Khalil, ${ }^{3,4,5}$ and W. Anis ${ }^{2}$ \\ ${ }^{1}$ The British University in Egypt (BUE), Cairo, Egypt \\ ${ }^{2}$ Ain Shams University, Cairo, Egypt \\ ${ }^{3}$ Cairo University, Giza, Egypt \\ ${ }^{4}$ Arab Academy for Science, Technology and Maritime Transport, Giza, Egypt \\ ${ }^{5}$ Fayoum University, Fayoum, Egypt
}

\begin{abstract}
In order to develop an optoelectronic model for simulating different light trapping structures sandwiching the photovoltaic active layer, determining the materials dispersion and absorption properties is a must. The targeted model should be able to simulate the desperation and absorption capabilities of different conductor and semiconductor materials over the entire sun spectrum $(200 \mathrm{~nm}$ to 1700 $\mathrm{nm}$ ). Therefore, the Lorentzian-Dude (LD) model is chosen due to its simplicity in implementation with the finite difference time domain algorithm chosen for optical modelling. In this paper, various materials are selected to be modelled with the LD model. The proposed algorithm is not only used for modelling material behaviour of various conducting materials published in literature, but is also used for other conducting and semiconducting materials that the original model was not capable of modelling over the entire range of spectrum. Besides that, the suggested algorithm showed a better time performance than those mentioned in literature. Experimental 1D grating structure prototype samples were made to validate the simulation results, showing perfect agreement.
\end{abstract}

Index Terms-FDTD, grating structures, Lorentzian-Dude (LD) model, Solar cells materials.

\section{INTRODUCTION}

In order to model dispersive and absorptive materials, the dispersion coefficients should be calculated. We use Lorentzian-Drude (LD) model as it could easily be incorporated into FDTD simulations [1]. The LD model is a combination of two models. The first one is the Drude model which effectively models conductivity, dispersion and background absorption due to electronic intraband transitions. The second one is the semi-quantum Lorentz model which describes electronic interband transitions. It is used for modelling dispersive media having multiple resonant frequencies (multipole dispersion). The complex permittivity of a material using LD model could be expressed by [1]:

$$
\varepsilon(\omega)=\varepsilon_{\bowtie}+\sum_{\mathrm{n}} \frac{\sigma_{\mathrm{n}} \omega_{\mathrm{n}}^{2}}{\omega_{\mathrm{n}}^{2}-\omega^{2}-\mathrm{i} \omega \Gamma_{\mathrm{n}}}
$$

where $\varepsilon_{\bowtie}$ is the instantaneous dielectric response (DC), $\Gamma_{\mathrm{n}}$ and $\omega_{\mathrm{n}}$ are the LD coefficients and $\sigma_{\mathrm{n}}$ is a func- tion of position specifying the strength of the $n^{\text {th }}$ resonance.

In this work, we enrolled the LD fitting algorithm in modelling the absorption and dispersion behaviour of various conducting as well as semiconducting materials used for solar cells. The algorithm was developed using Matlab scripting, where an optimization process took place for utilization and minimum time consumption. Then, MEEP (Massachusetts Institute for Technology Electromagnetic Equation Propagation) [2] is used as a simulation platform for the optical characterization of 1D grating structure.

\section{Modelling DisPersive CONDUCTING MATERIALS}

A fitting process takes place to calculate the LD coefficients of different materials, such as silver (Ag), indium tin oxide (ITO), gold $(\mathrm{Au})$, aluminium $(\mathrm{Al})$ and zinc oxide $(\mathrm{ZnO})$ within the solar spectrum from $200 \mathrm{~nm}$ to $1700 \mathrm{~nm}$. We have fitted the complex permittivity of the above 5 materials using equation (1). The fitting curves for these materials are shown in Figures 1 to 5 using the experimental data given in $[3,4,5]$, while the fitting parameters are listed in Table 1.

\section{Modelling DisPersive Semiconducting MATERIALS}

For $\mathrm{Si}$, no single fitting with Lorentz-Drude terms was found for both visible and near ultraviolet ranges [6]. Various trials were made to overcome such a problem; one of them was modelling only the visible range $(400 \mathrm{~nm}$ to $800 \mathrm{~nm}$ ) using three LD terms [7]. Another approach was proposed in [6] where modified LD model was introduced, making it possible to simulate the Si behaviour from $300 \mathrm{~nm}$ to $1000 \mathrm{~nm}$ using a single fit. In order to solve such a problem, we suggest a technique in [7], through modelling the behaviour of Si through the whole spectrum. This technique is based on portioning the spectrum into sub-bands and determining the LD terms for each sub-band individually, and then a concatenation process takes place. We will use the open-source implementation of the FDTD algorithm [2] MEEP as a simulation platform for the optical behaviour for the desired structures. 

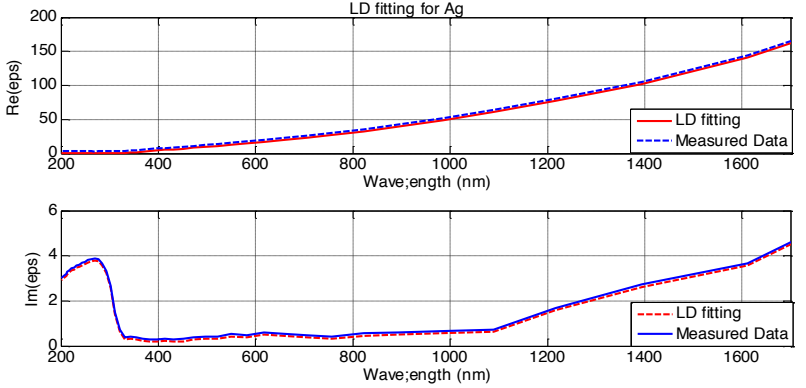

Figure 1. LD fitting curves for the real and the imaginary parts of the Ag permittivity using experimental data in [3].
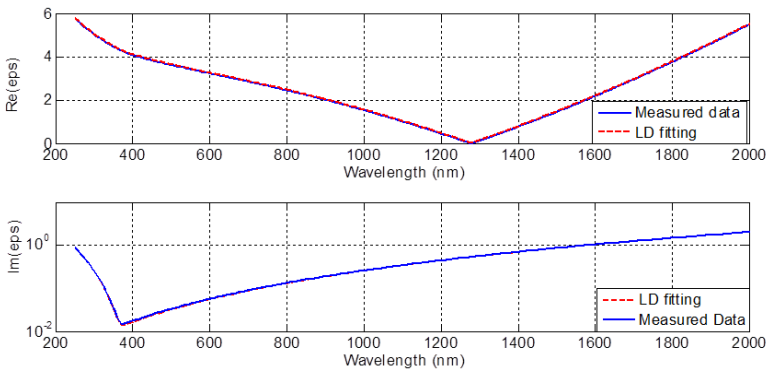

Figure 2. LD fitting curves for the real and the imaginary parts of the ITO permittivity using experimental data in [3].
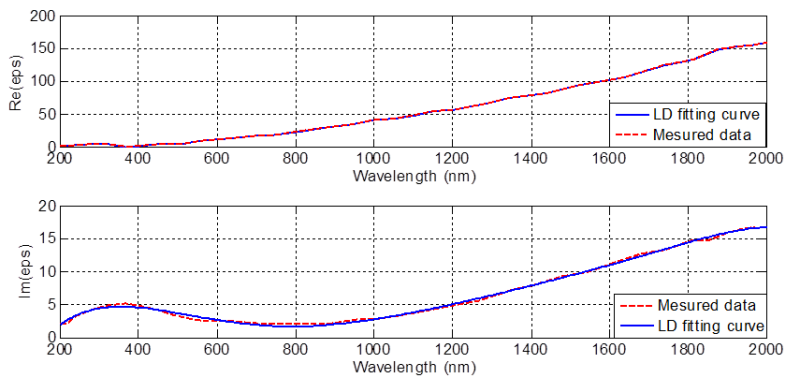

Figure 3. LD fitting curves for the real and the imaginary parts of the Au permittivity using experimental data in [3].
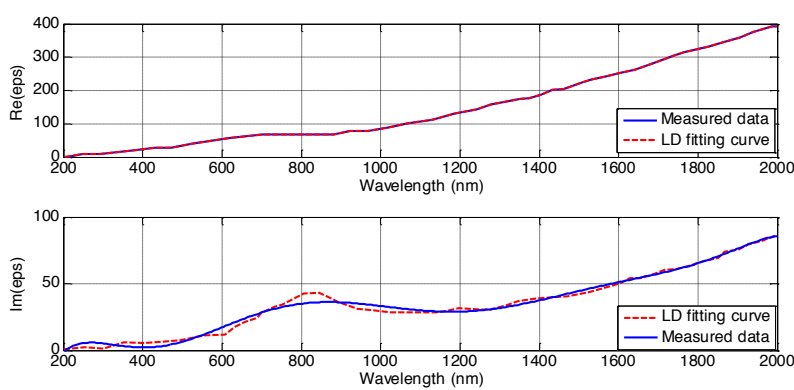

Figure 4. LD fitting curves for the real and the imaginary parts of the Al permittivity using experimental data in [4].
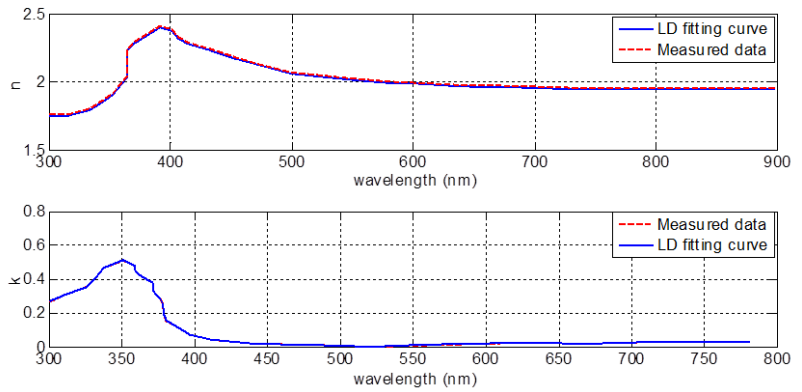

Figure 5. LD fitting curves for the real and the imaginary parts of the Al permittivity using experimental data in [5].
TABLE I.

LD FITTING PARAMETERS FOR AG, ITO, AU, AL AND ZNO

\begin{tabular}{|c|c|c|c|c|c|}
\hline & Ag & ITO & Au & Al & ZnO \\
\hline$\varepsilon_{\infty}$ & 1 & 1 & 1 & 1 & 1 \\
\hline$\sigma_{1}$ & $4.4625 \mathrm{e}+41$ & $5.1166 \mathrm{e}+40$ & $4.0314 \mathrm{e}+41$ & $7.6347 \mathrm{e}+41$ & $4.3873 \mathrm{e}+41$ \\
\hline$\omega_{1}$ & 0.83542 & 0.52365 & 0.0722 & 0.8935 & 0.4759 \\
\hline$\Gamma_{1}$ & 0.038715 & 0.066137 & 0.042747 & 0.037908 & 0.024197 \\
\hline$\sigma_{2}$ & 7.9247 & 79.136 & 0.27826 & 1941 & 84.489 \\
\hline$\omega_{2}$ & 0.65815 & 0.62669 & 0.33472 & 0.13066 & 0.23471 \\
\hline$\Gamma_{2}$ & 3.1343 & 1.8357 & 0.27826 & 0.26858 & 0.30488 \\
\hline$\sigma_{3}$ & 0.50133 & 8.7496 & 0.27826 & 4.7065 & 1.395 \\
\hline$\omega_{3}$ & 3.6142 & 1.2461 & 0.66944 & 1.2453 & 2.385 \\
\hline$\Gamma_{3}$ & 0.36456 & 2.0309 & 0.27826 & 0.25165 & 0.85172 \\
\hline$\sigma_{4}$ & 0.013329 & 1.5787 & 0.65677 & 11.396 & 3.0189 \\
\hline$\omega_{4}$ & 6.6017 & 2.0236 & 2.3947 & 1.4583 & 4.2747 \\
\hline$\Gamma_{4}$ & 0.052426 & 1.3413 & 0.7017 & 1.0897 & 2.5915 \\
\hline$\sigma_{5}$ & 0.82655 & 0.014077 & 2.6455 & 0.55813 & 0.59868 \\
\hline$\omega_{5}$ & 0.7388 & 1.5671 & 3.4714 & 2.8012 & 9.0173 \\
\hline$\Gamma_{5}$ & 0.7388 & 1.4211 & 2.0115 & 2.7278 & 3.4722 \\
\hline$\sigma_{6}$ & 1.1133 & & 2.0148 & & \\
\hline$\omega_{6}$ & 16.365 & & 10.743 & & \\
\hline$\Gamma_{6}$ & 1.9511 & & 1.7857 & & \\
\hline
\end{tabular}

For Si we divide the spectrum into three sub-bands. The first sub-band is from $200 \mathrm{~nm}$ to $400 \mathrm{~nm}$, the second subband is from $400 \mathrm{~nm}$ to $1000 \mathrm{~nm}$ and the third sub-band is from $1000 \mathrm{~nm}$ to $1700 \mathrm{~nm}$. Figures from 6 to 8 show a comparison between the experimentally measured permittivity [9] and the fitted LD model for the three sub-bands with the fitting parameters listed in table II. The fitting parameters are compared with previously determined ones in $[7,10]$ showing high accordance. To verify the method used, we calculate the mean error due to fitting process as a percentage from the measured data. The mean errors for both the real and imaginary parts of the Si permittivity are shown in figure 9.
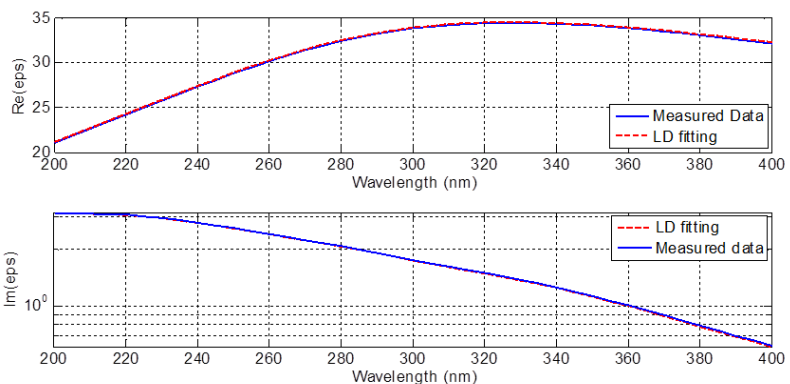

Figure 6. Experimental [9] and fitted Lorentz-Drude model for the real and imaginary parts of the silicon permittivity in the first sub-band where the imaginary part is plotted log scale. 

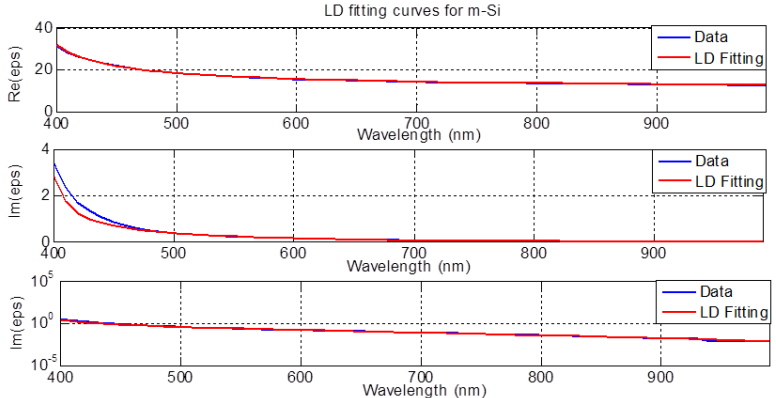

Figure 7. Experimental [9] and fitted Lorentz-Drude model for the real and imaginary parts of the silicon permittivity in the second sub-band where the imaginary part is plotted in both linear and log scales.
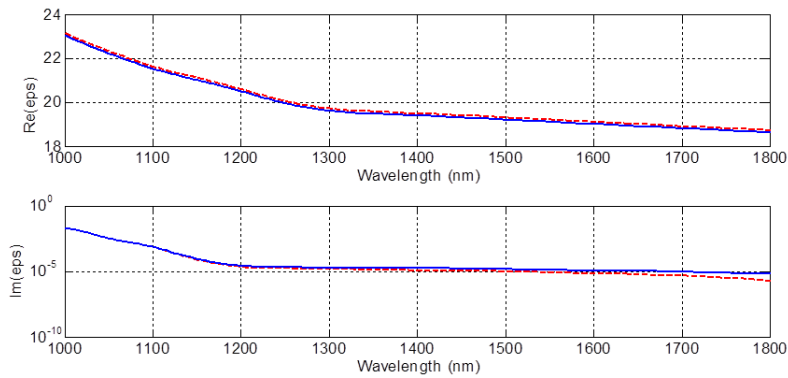

Figure 8. Experimental [9] and fitted Lorentz-Drude model for the real and imaginary parts of the silicon permittivity in the third sub-band where the imaginary part is plotted log scale.

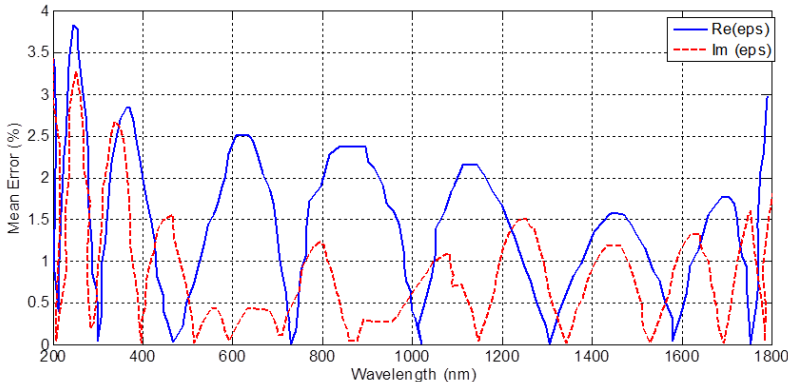

Figure 9. The mean error for the real and imaginary parts of Si permittivity through the entire wavelength of solar spectrum.

TABLE II.

LD FITTING PARAMETERS FOR SI IN THE THREE SUB-BANDS

\begin{tabular}{|c|c|c|c|}
\hline & First sub-band & Second sub-band & Third sub-band \\
\hline$\varepsilon_{\infty}$ & 1 & 1 & 1 \\
\hline$\sigma_{1}$ & $4.4625 \mathrm{e}+41$ & $5.1166 \mathrm{e}+40$ & $4.0314 \mathrm{e}+41$ \\
\hline$\omega_{1}$ & 0.83542 & 0.52365 & 0.0722 \\
\hline$\Gamma_{1}$ & 0.038715 & 0.066137 & 0.042747 \\
\hline$\sigma_{2}$ & 7.9247 & 79.136 & 0.27826 \\
\hline$\omega_{2}$ & 0.65815 & 0.62669 & 0.33472 \\
\hline$\Gamma_{2}$ & 3.1343 & 1.8357 & 0.27826 \\
\hline$\sigma_{3}$ & 0.50133 & 8.7496 & 0.27826 \\
\hline$\omega_{3}$ & 3.6142 & 1.2461 & 0.66944 \\
\hline$\Gamma_{3}$ & 0.36456 & 2.0309 & 0.27826 \\
\hline
\end{tabular}

To generalize the proposed approach, various semiconductor materials were simulated using the same approach. $\mathrm{Ge}$ is portioned into two sub-bands where fitting process takes place using data in [11] as shown in figure 10 and 11 for real and imaginary refractive index respectively. The LD parameters is then calculated and listed in Table III. Same process takes place for GaAs but with three subbands using data in [12] as shown in figure 12, 13 and 14 with LD coefficients listed in Table IV.
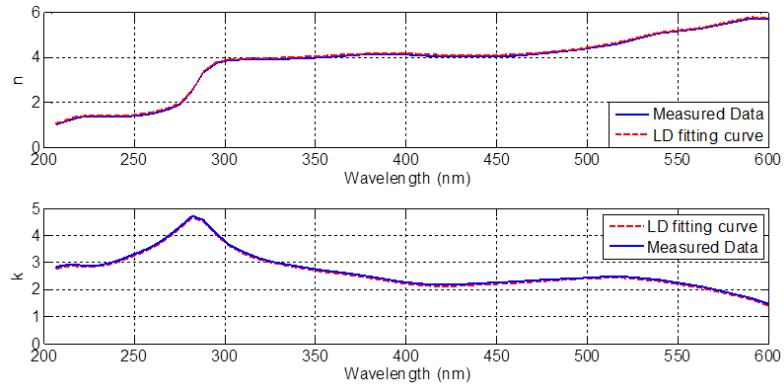

Figure 10. Experimental [11] and fitted Lorentz-Drude model for the real and imaginary parts of the Germanium refractive index in the first sub-band
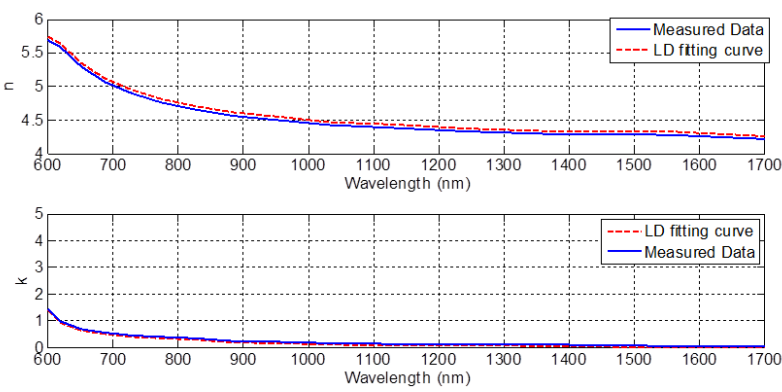

Figure 11. Experimental [11] and fitted Lorentz-Drude model for the real and imaginary parts of the Germanium refractive index in the second sub-band
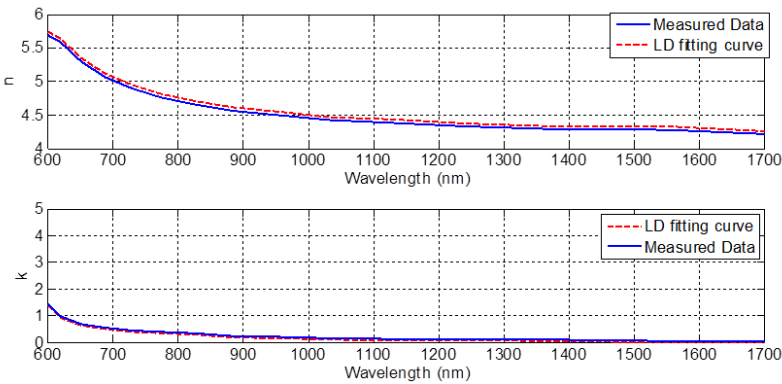

Figure 12. The mean error for the real and imaginary parts of Si permittivity through the entire wavelength of solar spectrum.
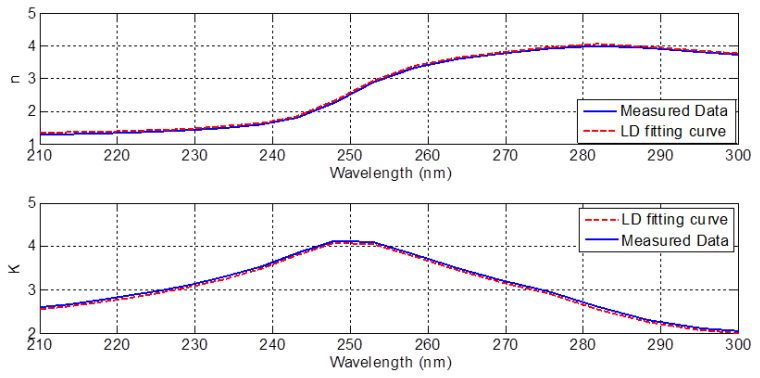

Figure 13. Experimental [12] and fitted Lorentz-Drude model for the real and imaginary parts of the GaAs refractive index in the second subband. 
TABLE III.

LD FITTING PARAMETERS FOR GE

\begin{tabular}{|c|c|c|}
\hline & First sub-band & Second sub-band \\
\hline$\varepsilon_{\infty}$ & 1 & 1 \\
\hline$\sigma_{1}$ & $4.4625 \mathrm{e}+41$ & $5.1166 \mathrm{e}+40$ \\
\hline$\omega_{1}$ & 0.83542 & 0.52365 \\
\hline$\Gamma_{1}$ & 0.038715 & 0.066137 \\
\hline$\sigma_{2}$ & 7.9247 & 79.136 \\
\hline$\omega_{2}$ & 0.65815 & 0.62669 \\
\hline$\Gamma_{2}$ & 3.1343 & 1.8357 \\
\hline$\sigma_{3}$ & 0.50133 & 8.7496 \\
\hline$\omega_{3}$ & 3.6142 & 1.2461 \\
\hline$\Gamma_{3}$ & 0.36456 & 2.0309 \\
\hline
\end{tabular}
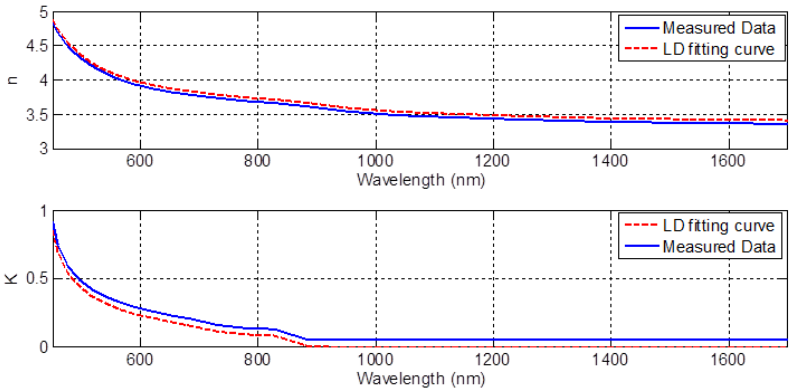

Figure 14. Experimental [12] and fitted Lorentz-Drude model for the real and imaginary parts of the GaAs refractive index in the third subband.

To check the utility of the new approach, a comparison between our suggested model (with variable number of LD coefficients) and the model suggest in [1] with fixed number of coefficients ( 5 coefficients) is shown for both the number of iteration needed and the simulation time in figures 15 and 16. The simulation is done on a core-i7 Intel processor with 16 GB RAM desktop computer. The comparison shows that our new suggested model outdoes that suggested in [1] as it achieves the total fitting required with lower number of iterations, giving the ability to tune the maximum accepted mean error. In addition to that, the model shows this utility for both metals and semiconductor materials.

\section{1D GRATING STRUCTURE}

We define a $1 \mathrm{D}$ grating as structures that are uniform in one dimension, e.g. y-direction, and are periodic in the other dimension (x-direction) with a periodicity $\mathrm{L}$ as shown in Figure 17a. We consider incident light propagating in the xz-plane. Since the structure considered is two dimensional, one can separately consider the response of the structures for the s-polarization, which has its electric field polarized along the $y$-direction, and for the $p$ polarization, which has its magnetic field polarized along the y-direction as shown in Figure 17b.

\section{Number of Iterations}

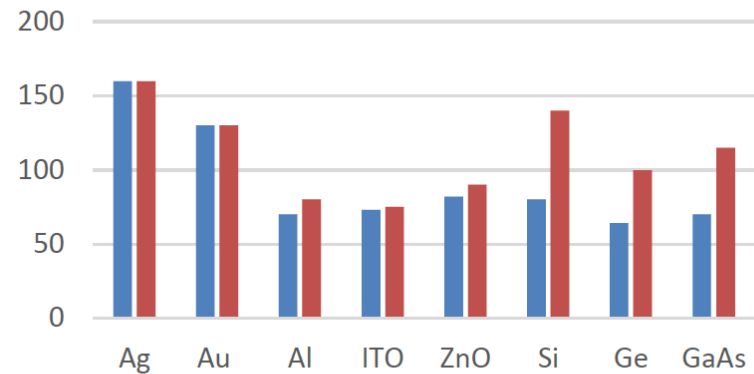

Number of iterations with variable LD cofficients

Number of iterations with 5 LD cofficients

Figure 15. The number of iterations required for variable and fixed LD coefficients.

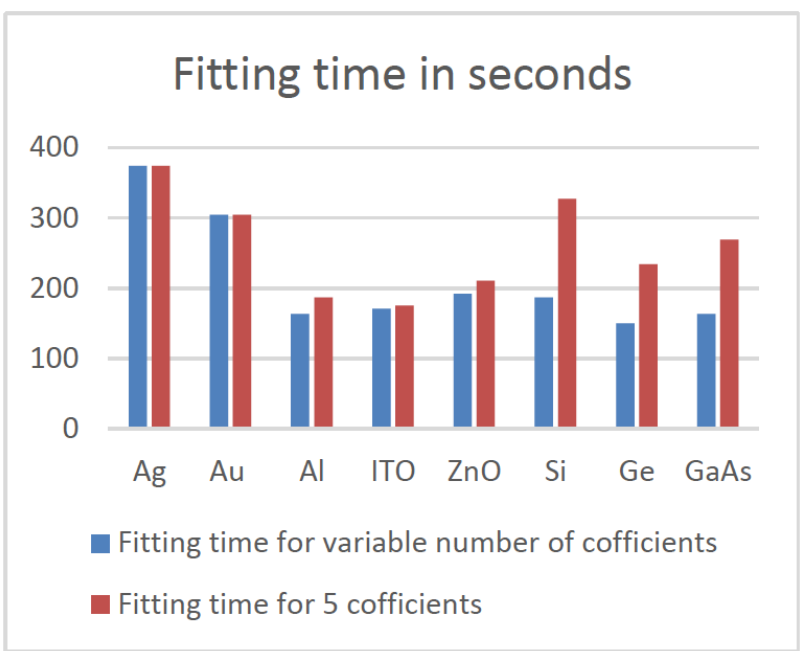

Figure 16. The fitting time required for variable and fixed LD coefficients.

A $1 \mathrm{D} \mathrm{Si} / \mathrm{SiO} 2$ grating structure is simulated with $\mathrm{SiO} 2$ thickness $\mathrm{d} 2$ of $100 \mathrm{~nm}$, a periodicity $\mathrm{L}$ of $1 \mu \mathrm{m}$ and an aspect ratio $(\mathrm{a} / \mathrm{b})$ of 1 (Figure $17 \mathrm{~b})$. The minimum dimension in the $\mathrm{x}$-direction is taken $\mathrm{b}=500 \mathrm{~nm}$ to make it possible to fabricate such a device on an area of $200 \mu \mathrm{m} \mathrm{x}$ $200 \mu \mathrm{m}$. The selected dimensions are far from the optimum one for an optical band gap in the desired spectrum portion with high reflectivity. However, this structure will be useful to validate our numerical simulation model as it contains a lot of peaks and troughs making it challenging to compare the numerical simulation results with the experimental one and this is consider as a future work. Another trial was made to simulate the same structure but with aspect ratio 2.4. The reflectance of this structure is shown in Figure 18 with an optical band gap in the near infrared region. 


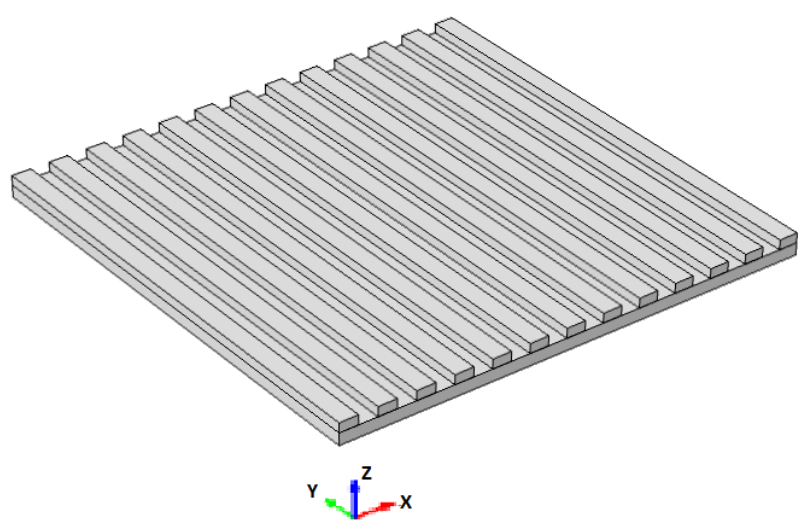

(a)

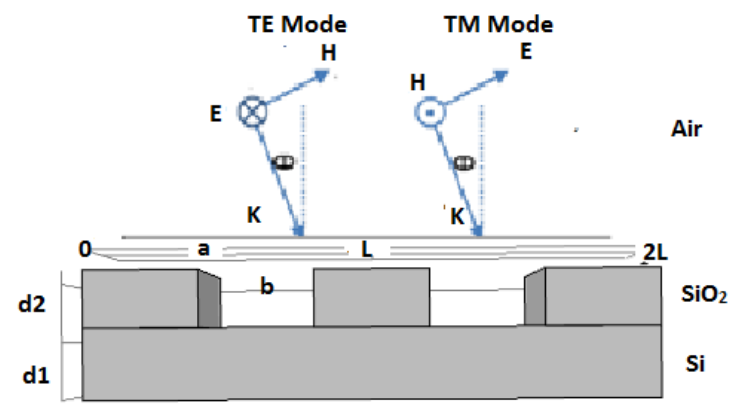

$\downarrow$ (b)

Figure 17. a) 3D schematic for the $1 \mathrm{D}$ grating strucures. (b) $\mathrm{X}-\mathrm{Z}$ cross section for $1 \mathrm{D}$ grating structure

\section{CONCLUSION}

This paper introduces a new technique for modeling dispersive materials across the ultra-violet, visible and near infrared regions using the MEEP simulation tool, which relies on solving Maxwell's equations using the FDTD technique. Our proposed technique is unique, since it enables the simulations community to model the propagation of light through materials such as Si through the entire Sun's wavelength spectrum. A 1D grating structure is then simulated to verify the utility of the developed model.

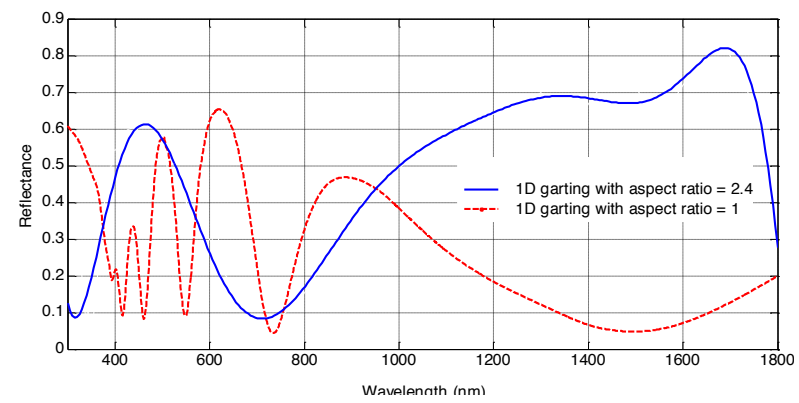

Figure 18. 1D grating structure with aspect ratio of 1 (red curve) and 2.4 (blue curve)

\section{ACKNOWLEDGEMENTS}

This work is part of SURSYS project funded by the German Academic Exchange Service (DAAD) and financed by the Federal Foreign Office. The authors acknowledge the valuable support of Prof. Mathias U1bricht, Dr. Michael Eisinger and Mr. Simon Kustos (Kresmann). We would also like to acknowledge the sup- port of The World Academy of Sciences (TWAS) for providing the necessary computational resources.

\section{REFERENCES}

[1] A. D. Rakić, A. B. Djurišić, J. M. Elazar, and M. L. Majewski, "Optical properties of metallic films for vertical-cavity optoelectronic devices," Applied Optics, vol. 37, no. 22, pp. 5271-5283, 1998. https://doi.org/10.1364/AO.37.005271

[2] J. D. Joannopoulos, "MEEP," Massachusetts Institute of Technology, 3 October 2012. [Online]. Available: http://abinitio.mit.edu/wiki/index.php/Meep. [Accessed 1 March 2013].

[3] T. Marie-Luce , "Investigation of the optical properties of Au by Means of Thin Semitransparent Films," Physics Review B, vol. B, no. 2, pp. 3060-3078, 1970.

[4] A. D. Rakić , "Algorithm for the determination of intrinsic optical constants of metal films: application to aluminum," Applied Optics , vol. 34, pp. 4755-4767, 1995. https://doi.org/10.1364/AO.34. $\underline{004755}$

[5] D. Zhong-Hong, Z. Rong-Jun, S. Jie, C. Yi-Ming, Z. Yu-Xiang, W. Jia-Da and C. Liang-Yao, "Optical Properties of Zinc-oxide Films Determined Using Spectroscopic," Journal of the Korean Physical Society, vol. 55, no. 3, pp. 1227-1232, 2009. https://doi.org/10.3938/jkps.55.1227

[6] D. Alexei and J. Sajeev, "Effective optical response of silicon to sunlight in the finite-difference time-domain method," Optics letter, vol. 37, no. 1, pp. 112-114, 2012.

[7] A. Deinega, I. Valuev, B. Potapkin and Y. Lozovik, "Minimizing light reflection from dielectric textured surfaces," The Journal of the Optical Society of America A, vol. 25, no. 5, pp. 770-777, 2011. https://doi.org/10.1364/JOSAA.28.000770

[8] S. Abdellatif, R. Ghannam and A. S. G. Khalil, "Simulating the dispersive behavior of semiconductors using the LorentzianDrude model for photovoltaic devices," Applied Optics 53, 32943300 (2014). https://doi.org/10.1364/AO.53.003294

[9] J. H. Michael and J. H. Stephen, "Optical Properties of Silicon," Virginia Semiconductor, Inc., Fredericksburg, 2000.

[10] M. Mishrikey, "Analysis and Design of Metamaterials," SWISS FEDERAL INSTITUTE OF TECHNOLOGY , ZURICH, 2010.

[11] A. H. Clark, "Electrical and Optical Properties of Amorphous Germanium," Physics Review, vol. 154, p. 750-757, 1967. https://doi.org/10.1103/PhysRev.154.750

[12] J. S. Blakemore, "Semiconducting and other major properties of gallium arsenide," Journal of Applied Physics , vol. 53, no. 10, pp. R132-R181, 1982. https://doi.org/10.1063/1.331665

\section{AUTHORS}

S. Abdellatif is with The British University in Egypt (BUE), Cairo, Egypt (sameh.osama@bue.edu.eg).

K. Kirah is with the Faculty of Engineering, Ain Shams University, Cairo, Egypt (khaled.kirah@eng.asu. edu.eg).

R. Ghannam is with Egypt Nanotechnology Research Center, Cairo University, Giza, Egypt (rmghannam@, gmail.com)

A. S. G. Khalil is with Egypt Nanotechnology Research Center, Cairo University, Giza, Egypt, with Arab Academy for Science, Technology and Maritime Transport, Smart Village Campus, Giza, Egypt, and with the Center for Environmental and Smart Technology, Faculty of Science, Fayoum University, Fayoum, Egypt (ahmed.s.g.khalil@uni-due.de).

W. Anis is with the Faculty of Engineering, Ain Shams University, Cairo, Egypt (wagdy4451@yahoo.com).

Submitted 25 October 2016. Published as resubmitted by the authors 27 November 2016. 